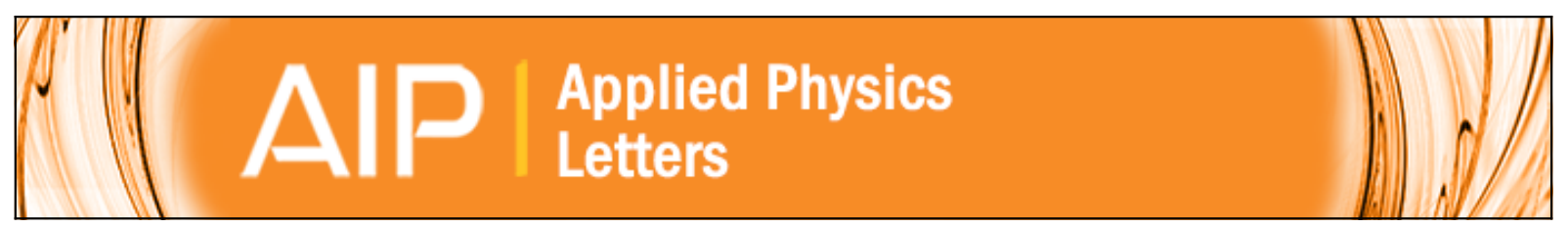

Evidence for critical scaling of plasmonic modes at the percolation threshold in metallic nanostructures

Eser Metin Akinoglu, Tianyi Sun, Jinwei Gao, Michael Giersig, Zhifeng Ren, and Krzysztof Kempa

Citation: Applied Physics Letters 103, 171106 (2013); doi: 10.1063/1.4826535

View online: http://dx.doi.org/10.1063/1.4826535

View Table of Contents: http://scitation.aip.org/content/aip/journal/apl/103/17?ver=pdfcov

Published by the AIP Publishing 


\title{
Evidence for critical scaling of plasmonic modes at the percolation threshold in metallic nanostructures
}

\author{
Eser Metin Akinoglu, ${ }^{1, a)}$ Tianyi Sun, ${ }^{2,3, a)}$ Jinwei Gao, ${ }^{4}$ Michael Giersig, ${ }^{1, b)}$ Zhifeng Ren, ${ }^{3, b)}$ \\ and Krzysztof Kempa ${ }^{2, b)}$ \\ ${ }^{1}$ Department of Physics, Freie Universität Berlin, 14195 Berlin, Germany \\ ${ }^{2}$ Department of Physics, Boston College, Chestnut Hill, Massachusetts 02467, USA \\ ${ }^{3}$ Department of Physics and TcSUH, University of Houston, Houston, Texas 77204, USA \\ ${ }^{4}$ Institute for Advanced Materials (IAM), Academy of Advanced Optoelectronics, South China Normal \\ University, Guangzhou 510006, People's Republic of China
}

(Received 7 September 2013; accepted 7 October 2013; published online 22 October 2013)

\begin{abstract}
In this work we provide the experimental demonstration of critical scaling of plasmonic resonances in a percolation series of periodic structures which evolve from arrays of holes to arrays of quasi-triangles. Our observations are in agreement with the general percolation theory and could lead to sensor and detector applications. (C) 2013 AIP Publishing LLC. [http://dx.doi.org/10.1063/1.4826535]
\end{abstract}

Metamaterial design as well as sensor and detector applications require systems with high sensitivity of physical phenomena to subtle changes in system parameters. Such sensitivity occurs in percolation systems, made of planar arrays of metallic islands or holes in thin metallic films,${ }^{1-7}$ both commonly employed in different plasmonic and metamaterial structures. These topologically distinct pattern systems have vastly different in-plane electronic conductivities that change from large in the array of holes to vanishingly small in the island array, just above the percolation threshold (PT). Percolation problems, which closely resemble second-order phase transitions, ${ }^{8,9}$ are characterized by power law dependencies on the metallic island area fraction $p$ and on the frequency $\omega$ when the critical area fraction $p_{c}$ is approached. For composite systems consisting of highly conducting particles in a dielectric matrix, the following relations have been predicted and observed at low electromagnetic frequencies. ${ }^{10-13}$ At the PT, where $p=p_{c}$, the following power laws are obeyed by the effective conductivity $\left(\sigma_{e f f}\right)$

$$
\sigma_{e f f} \sim \omega^{\beta},
$$

and the effective dielectric function

$$
\varepsilon_{\text {eff }} \sim \omega^{\beta-1}
$$

where $0<\beta<1$ is the critical exponent. Near the PT, both the conductivity and dielectric function are given by power laws in terms of $\left(p-p_{c}\right)$. In particular, for $p>p_{c}$

$$
\sigma_{e f f} \sim\left(p-p_{c}\right)^{\gamma \beta},
$$

and $p<p_{c}$

$$
\varepsilon_{e f f} \sim\left(p_{c}-p\right)^{\gamma(\beta-1)},
$$

where $\gamma>0$ is another critical exponent. A special case of the percolation problem is the periodic percolation series, in

\footnotetext{
${ }^{\text {a) }}$ E. M. Akinoglu and T. Sun contributed equally to this work.

${ }^{b)}$ Authors to whom correspondence should be addressed. Electronic addresses: kempa@bc.edu; giersig@physik.fu-berlin.de; and zren@uh.edu
}

which fixed hole locations form a periodic lattice. As a result of the Babinet principle, ${ }^{14}$ the electromagnetic response spectra for a percolation series, evolving from hole to island arrays, are closely related. ${ }^{15-18} \mathrm{~A}$ very high symmetry periodic percolation series is obtained with square holes (square periodic percolation series, SPPS), where the patterns on opposite sides of the percolation threshold form complementary Babinet pairs. ${ }^{18}$ In this work, we employ a simple and easy technique to manufacture periodic percolation series based on a hexagonal array of circular holes (circular periodic percolation series, CPPS). ${ }^{16}$ This special example of the percolation problem represents a case of a discontinuous percolation transition, ${ }^{9}$ extensively studied in the context of explosive percolation, ${ }^{19}$ with an instantaneously expanding correlation length $\zeta$, the length scale that determines the size of the cluster of connected islands, at the PT. This implies that $\zeta \sim\left(p_{c}-p\right)^{-\nu}$ for $p<p_{c}$, with the critical exponent $\nu \rightarrow 0$ and that the DC effective conductivity vanishes according to $\sigma_{\text {eff }} \sim\left(p-p_{c}\right)$ for $p>p_{c},{ }^{9}$ which implies with use of Eq. (3) that $\gamma \beta=1$. Critical behavior consistent with these dependencies has been confirmed theoretically for SPPS ${ }^{18}$ and via simulations for CPPS. ${ }^{16}$ Here, we demonstrate the universal critical behavior of spectra in the high frequency plasmonic range in CPPS near the percolation threshold by studying experimentally the transmittance of light in the infrared (IR) and visible ranges. Additionally, we show radio frequency conductivity measurements in selected CPPS structures. This direct experimental evidence is supported by finite difference time domain (FDTD) simulations and is consistent with the general percolation theory.

Our CPPS is obtained by producing hexagonal array patterns of circular holes of increasing diameter in the subsequent structures, made in a thin bimetallic film consisting of $50 \mathrm{~nm}$ plasmon-active gold on a $3 \mathrm{~nm}$ titanium adhesion layer on a sapphire substrate. A total of 13 patterns were made by employing the nanosphere lithography (NSL) technique, also known as the natural lithography or the colloidal lithography, ${ }^{20-24}$ including 6 in the immediate vicinity of the percolation threshold. Scanning electron microscope (SEM) micrographs were gathered on a Hitachi SU8030 SEM, and a few examples at the percolation threshold are shown in 

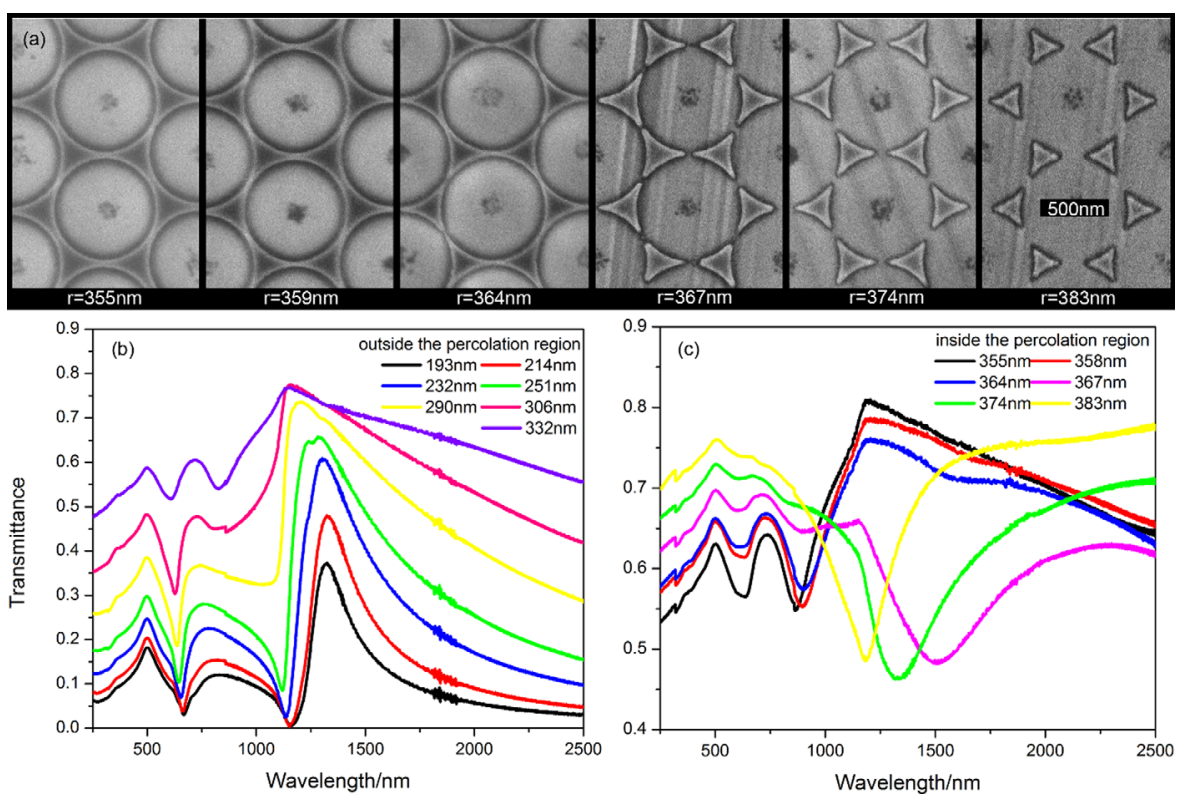

FIG. 1. (a) SEM micrographs of selected CPPS patterns transforming from metallic hole to island arrays. Plots of the measured transmittance vs radiation wavelength for arrays below the percolation threshold (b) and in the percolation region (c).
Figure 1(a). Patterns change gradually from hexagonal arrays of circular holes of increasing diameter to honeycomb arrays of disconnected quasi-triangular metallic islands.

We focus on the normal angle optical transmittance $T$ through these patterned structures as the main probe of the electromagnetic response. The complete set of the transmittance spectra, collected with a Perkin-Elmer Lambda 950 spectrophotometer, are shown in Figure 1(b), outside the percolation region and (c) inside the region. In the percolation region, these data are also displayed as an color-encoded map of transmittance versus wavelength and hole radius in Figure 2(a). The color-encoded map of the corresponding numerical simulations is shown in Figure 2(b). The simulations were performed with MIT Electromagnetic Equation Propagation (MEEP) 1.1.1, which is a finite-difference timedomain (FDTD) simulation software package widely used in the field. ${ }^{25,26}$ The permittivity of gold is fitted to the LorentzDrude model, ${ }^{27}$ and that for the sapphire was set to 3.2 . We have made a slight adjustment $(<1 \%)$ of $r^{*}$ in simulations to align with experiment. This is justified by the finite numerical mesh size, comparable to the adjustment. The dashed lines representing minima of $\mathrm{T}$, in experimental panel (a), are obtained from the simulation panel (b), and those in the simulation panel (b) are obtained from the experimental panel (a).

On the hole side of CPPS, the spectra are dominated by a series of peaks separated by deep minima. FDTD simulations are in a broad and qualitative agreement with all experimental features, in particular reproducing the rapid shift of the deepest minimum, highlighted as a green band in Figure 2(a), on the approach to the critical hole radius $r^{*} \approx 365 \mathrm{~nm}$. The observed transmission maxima correspond to the wellknown extraordinary optical transmission (EOT) effect, mediated by surface plasmon polaritons and enabled by the periodic nature of the CPPS, ${ }^{6,28}$ whereas the main minima correspond to plasmonic resonances trapping radiation in the film. The characteristic reversal of the behavior is observed
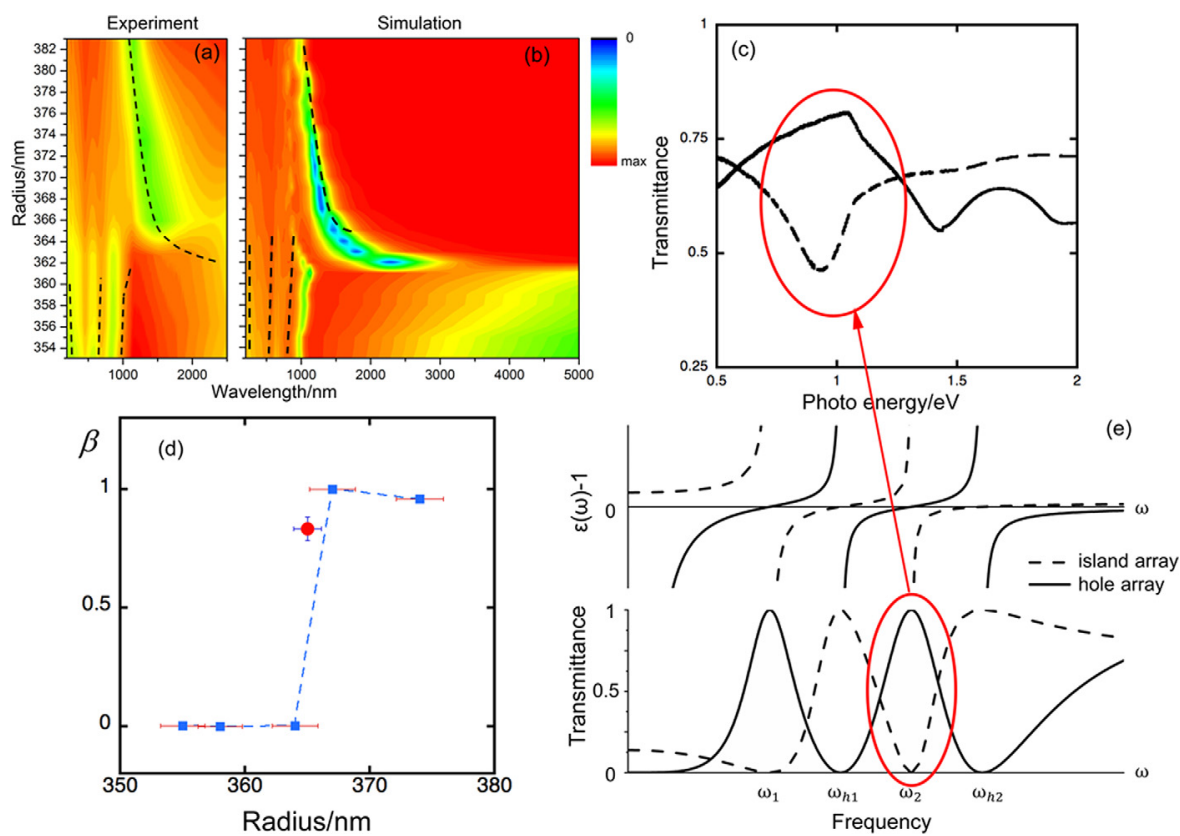

FIG. 2. Color maps of the transmittance of CPPS versus wavelength and the hole size: experiment (a) and simulation (b). The dashed lines outlining minima locations are guides to the eye: those in (a) outline minima locations of simulation in (b) and those in (b) outline minima locations of the experiments in (a). (c) Plots of the transmittance vs photon energy for two CPPS with $r=355 \mathrm{~nm}$ (solid line) and $r=374 \mathrm{~nm} \quad$ (dashed line). (d) $\beta$ extracted from the $\ln \left(\sigma_{\text {eff }}\right)$ vs $\ln (\omega)$ slopes (blue squares) and $\beta$ extracted from the shift of the plasmonic resonance shown in Figures 2(a) and 2(b) (the red-solid circle). (e) Sketch of the effective dielectric function for the CPPS (upper panel), and the corresponding effective transmittance (lower panel). 
on the island side of the CPPS, ${ }^{15-18}$ with the main maxima becoming minima and vice versa (Figure 2(c)). The evolution of the transmittance spectra in CPPS reveals the expected critical behavior at the percolation threshold. The location of the first minimum on the island side of CPPS satisfies the power law

$$
\omega_{1} \sim\left(r-r^{*}\right)^{\zeta}
$$

The least-square-fit-extracted critical exponent is $\zeta=0.1$.

A simple effective medium approach allows for a basic understanding of the spectra (Figures 2(a)-2(c)). For the chosen perpendicular propagation direction, island films can be viewed as an effective medium, with a dielectric function for a lossless metal, given in its simplest form by

$$
\varepsilon(\omega)=1+\sum_{i} \frac{\omega_{p i}^{2}}{\omega_{i}^{2}-\omega^{2}},
$$

where $\omega$ is the frequency of the electromagnetic radiation, and $\omega_{i} \neq 0$ are the plasmonic (Mie) resonances in the islands. ${ }^{29}$ The dielectric function given by Eq. (6) is sketched out as lines in Figure 2(e), top. Here, all solid lines represent hole arrays, and all dashed lines represent island arrays. It can be shown ${ }^{6,16-18}$ that the dielectric function for hole arrays is also given by Eq. (6), but with different constants. In general, one of the resonant frequencies (say, one for $i=1$ ) vanishes $\left(\omega_{1}=0\right)$, leading to a divergent Drude-like term for vanishing frequency as shown by the solid line. For a hole array, strictly Babinet complementary to an island pattern, the spectrum is shifted ${ }^{18}$ so that the poles of $\varepsilon(\omega)$ for the hole array occur at the frequency locations where $\varepsilon(\omega)=1$ for the island array and vice-versa. This property can be expected only approximately for non-Babinet complementary pattern pairs, which is the case for our CPPS. The properties of $\varepsilon(\omega)$ imply the corresponding behavior of transmittance via the formula

$$
T=\frac{1}{1+\alpha^{2} \omega^{2}[1-\varepsilon(\omega)]^{2}},
$$

where $\alpha=d / 2 c\left(\alpha^{2} \omega^{2} \ll 1\right), d$ is the metallic film thickness, and $c$ is the speed of light. ${ }^{30}$ An immediate consequence and obvious result of this formula is that $T=1$ for $\varepsilon(\omega)=1$, and because of the smallness of $\alpha^{2} \omega^{2} \ll 1, T \approx 1$ for finite $\varepsilon(\omega)$. This is an alternative effective medium interpretation of EOT. ${ }^{6}$ On the other hand, $T$ has deep minima only at the poles of $\varepsilon(\omega)$, i.e., at plasmon (Mie) resonances. This immediately explains the $T$ dependencies qualitatively for island and hole arrays (Figure 2(e), bottom). As the holes transform into islands at the percolation threshold $r=r^{*} \approx 365 \mathrm{~nm}$, the features of maxima turn expectedly into minima (Figure 2(c)). Even though the real structures employ gold, a noble metal that, at optical frequencies, exhibits a high conductivity with a small loss factor as determined by the imaginary part of the metal's permittivity and the hole sizes are comparable or larger than the probing light wavelength, violating the long wavelength limit which is normally required for the effective medium approaches, this qualitative picture holds for the measured as well as simulated spectra of $T$ (Figures 2(a) and 2(b)). ${ }^{18}$
The power law given by Eq. (5) can be easily derived from Eq. (6), which yields $\varepsilon_{e f f}=1+\omega_{p l}^{2} / \omega_{1}^{2} \sim \omega_{1}^{-2}$ for a sufficiently small frequency, a nonzero $\omega_{1}^{2} \ll \omega_{p l}^{2}$ and $\omega_{1}^{2} \ll \omega_{i}^{2}$. From this scaling, the fact that $r \sim p$ and $r^{*} \sim p_{c}$, and from Eq. (4), we obtain Eq. (5) with the critical exponent $\zeta=-0.5 \gamma(\beta-1)$. Since for CPPS $\gamma \beta=1$, and also $\zeta=0.1$, the universal critical exponent $\beta=0.83$. This value is comparable of typical values, such as $\beta=0.86-0.92$ extracted recently from experiments with $3 \mathrm{D}$ composites. ${ }^{13}$ The critical exponent $\beta$ value for strictly Babinet complementary SPPS has been determined as $\beta=0.5{ }^{18}$ Similar results were extracted from random $2 \mathrm{D}$ resistor networks. ${ }^{31}$ The value of $\beta$ extracted from the plasmonic resonance power law in Eq. (5) is shown in Figure 2(d) as a red-solid circle, and its location is consistent with the low frequency results (blue squares). These results were obtained by measuring $\sigma_{\text {eff }}$ versus $\omega$ with an Agilent E4980A precision LCR meter in the frequency range from $20 \mathrm{~Hz}$ to $2 \mathrm{MHz}$. To facilitate these measurements, $120 \mathrm{~nm}$ thick silver contact electrodes were deposited onto the CPPS samples by magnetron sputtering (AJA international). The $\beta$ values extracted by fitting the measured $\sigma_{\text {eff }}$ vs. $\omega$ to Eq. (1) are shown as blue squares in Figure 2(d).

It is clear from Figure 1(c) that at the percolation threshold ( $365 \mathrm{~nm}$ here) there is a large, easily detectable change of transmittance of the structure: note that at $\lambda=1500 \mathrm{~nm}$ there is an easily detectable, nearly $30 \%$ change in $T$ when $r$ changes by less than $1 \%$ (from $364 \mathrm{~nm}$ to $367 \mathrm{~nm}$ ). This is because the maximum of $T$ just below PT turns into minimum immediately above. This strong sensitivity of response at the percolation threshold in the CPPS could be exploited in the design of various metamaterial systems of a desired response as well as in detector applications. This can be obtained by slight modifications of $r$ at the percolation threshold, for example, by compression, temperature variation, defect formation due to adsorbed biological molecules, etc. Thus, various plasmonic and/or metamaterial sensor or detection systems could be built based on this critical phenomenon.

In conclusion, we have demonstrated critical behavior of a periodic percolation series of planar structures evolving from arrays of holes to islands; the evolution being driven by the increasing of hole size. We show that in addition to low frequency (radio) conductivity, which shows the well-known power law dependency on the frequency, the high frequency (IR) location of plasmonic resonances exhibits also a power law dependency on the hole size around the percolation threshold. This phenomenon could be useful in various metamaterial designs and detector applications.

The work performed at Boston College was supported in part by the W. M. Keck Foundation Grant. M.G. thanks Helmholtz Zentrum Berlin, Poznan University (UAM) and Foundation for Polish Science in Warsaw for financial support. The work performed at University of Houston was funded by the US Department of Energy under Contract No. DOE DE-FG02-13ER46917. The authors thank Dr. K. Ellmer for helping with the evaporation of gold and Dr. A. J. Morfa for helping with the preparation of the manuscript. 
${ }^{1}$ F. J. G. de Abajo, Rev. Mod. Phys. 79, 1267 (2007).

${ }^{2}$ A. Sánchez-Iglesias, I. Pastoriza-Santos, J. Pérez-Juste, B. RodríguezGonzález, F. J. G. de Abajo, and L. M. Liz-Marzán, Adv. Mater. 18, 2529 (2006).

${ }^{3}$ J. Nelayah, L. Gu, W. Sigle, C. T. Koch, I. Pastoriza-Santos, L. M. Liz-Marzán, and P. A. Van Aken, Opt. Lett. 34, 1003 (2009).

${ }^{4}$ Y. Peng, C. Marcoux, P. Patoka, M. Hilgendorff, M. Giersig, and K. Kempa, Appl. Phys. Lett. 96, 133104 (2010).

${ }^{5}$ F. J. Garcia-Vidal, L. Martin-Moreno, T. W. Ebbesen, and L. Kuipers, Rev. Mod. Phys. 82, 729 (2010).

${ }^{6}$ W.-C. Chen, N. I. Landy, K. Kempa, and W. J. Padilla, Adv. Opt. Mater. 1, 221 (2013).

${ }^{7}$ Y. Wang, E. W. Plummer, and K. Kempa, Adv. Phys. 60, 799 (2011).

${ }^{8}$ D. Stauffer and A. Aharony, Introduction to Percolation Theory (Taylor \& Francis, London, UK, 1991).

${ }^{9}$ M. Sahimi, Applications of Percolation Theory (Taylor \& Francis, London, UK, 1994); M. Sahimi, B. D. Hughes, L. E. Scriven, and H. T. Davis, J. Phys. C 16, L521 (1983).

${ }^{10}$ A. L. Efros and B. I. Shklovskii, Phys. Status Solidi B 76, 475 (1976).

${ }^{11}$ D. J. Bergman and Y. Imry, Phys. Rev. Lett. 39, 1222 (1977).

${ }^{12}$ D. M. Grannan, J. C. Garland, and D. B. Tanner, Phys. Rev. Lett. 46, 375 (1981).

${ }^{13}$ R.-F. Wu, W. Pan, S.-L. Shi, and R.-B. Han, J. Appl. Phys. 102, 056104 (2007).

${ }^{14}$ J. D. Jackson, Classical Electrodynamics (Wiley, New York, USA, 1998).

${ }^{15}$ H.-T. Chen, J. F. O'Hara, A. J. Taylor, R. D. Averitt, C. Highstrete, M. Lee, and W. J. Padilla, Opt. Express 15, 1084 (2007).
${ }^{16}$ Y. Peng, T. Paudel, W.-C. Chen, W. J. Padilla, Z. F. Ren, and K. Kempa, Appl. Phys. Lett. 97, 041901 (2010).

${ }^{17}$ T. Sun, E. M. Akinoglu, C. Guo, T. Paudel, J. Gao, Y. Wang, M. Giersig, Z. Ren, and K. Kempa, Appl. Phys. Lett. 102, 101114 (2013).

${ }^{18}$ K. Kempa, Phys. Status Solidi (RRL) 4, 218 (2010).

${ }^{19}$ D. Achlioptas, R. M. D'Souza, and J. Spencer, Science 323, 1453 (2009); O. Riordan and L. Warnke, Science 333, 322 (2011); Y. S. Cho, S. Hwang, H. J. Herrmann, and B. Kahng, Science 339, 1185 (2013).

${ }^{20}$ N. Denkov, O. Velev, P. Kralchevski, I. Ivanov, H. Yoshimura, and K. Nagayama, Langmuir 8, 3183(1992).

${ }^{21}$ M. Giersig and P. Mulvaney, Langmuir 9, 3408 (1993).

${ }^{22}$ J. C. Hulteen and R. P. Van Duyne, J. Vac. Sci. Technol. A 13, 1553 (1995).

${ }^{23}$ A. Kosiorek, W. Kandulski, P. Chudzinski, K. Kempa, and M. Giersig, Nano Lett. 4, 1359 (2004).

${ }^{24}$ P. Patoka and M. Giersig, J. Mater. Chem. 21, 16783 (2011).

${ }^{25}$ A. F. Oskooi, D. Roundy, M. Ibanescu, P. Bermel, J. D. Joannopoulos, and S. G. Johnson, Comput. Phys. Commun. 181, 687 (2010).

${ }^{26}$ A. Taflove and S. C. Hagness, Computational Electrodynamics: The Finite-Difference Time-Domain Method (Artech House, Norwood, MA, USA, 2005).

${ }^{27}$ A. D. Rakic, A. B. Djurišic, J. M. Elazar, and M. L. Majewski, Appl. Opt. 37, 5271 (1998).

${ }^{28}$ T. W. Ebbesen, H. J. Lezec, H. F. Ghaemi, T. Thio, and P. A. Wolff, Nature 391, 667 (1998).

${ }^{29}$ K. Kempa, Phys. Rev. B 74, 033411 (2006).

${ }^{30}$ O. S. Heavens, Optical Properties of Thin Solid Films (Dover Publications, Inc., New York, USA, 1965).

${ }^{31}$ I. Webman, J. Jortner, and M. H. Cohen, Phys. Rev. B 16, 2593 (1977). 